\title{
Gut-derived uremic toxin handling in vivo requires OAT-mediated tubular secretion in chronic kidney disease
}

\author{
Kevin T. Bush, ${ }^{1}$ Prabhleen Singh, ${ }^{2,3}$ and Sanjay K. Nigam ${ }^{1,3}$ \\ 'Department of Pediatrics, University of California, San Diego, La Jolla, California, USA. 'Division of Nephrology- \\ Hypertension, University of California, San Diego and Veterans Affairs San Diego Healthcare System, San Diego, \\ California, USA. ${ }^{3}$ Department of Medicine, University of California, San Diego, La Jolla, California, USA.
}

\begin{abstract}
The role of the renal organic anion transporters OAT1 (also known as SLC22A6, originally identified as NKT) and OAT3 (also known as SLC22A8) in chronic kidney disease (CKD) remains poorly understood. This is particularly so from the viewpoint of residual proximal tubular secretion, a key adaptive mechanism to deal with protein-bound uremic toxins in CKD. Using the subtotal nephrectomy (STN) model, plasma metabolites accumulating in STN rats treated with and without the OAT inhibitor, probenecid, were identified. Comparisons with metabolomics data from Oat1$\mathrm{KO}$ and Oat3-KO mice support the centrality of the OATs in residual tubular secretion of uremic solutes, such as indoxyl sulfate, kynurenate, and anthranilate. Overlapping our data with those of published metabolomics data regarding gut microbiome-derived uremic solutes - which can have dual roles in signaling and toxicity - indicates that OATs play a critical role in determining their plasma levels in CKD. Thus, the OATs, along with other SLC and ABC drug transporters, are critical to the movement of uremic solutes across tissues and into various body fluids, consistent with the remote sensing and signaling theory. The data support a role for OATs in modulating remote interorganismal and interorgan communication (gut microbiota-blood-liver-kidney-urine). The results also have implications for understanding drug-metabolite interactions involving uremic toxins.
\end{abstract}

Conflict of interest: The authors have declared that no conflict of interest exists.

Copyright: () 2020, American Society for Clinical Investigation.

Submitted: September 27, 2019 Accepted: February 28, 2020 Published: April 9, 2020.

Reference information: /CI Insight. 2020;5(7):e133817.

https://doi.org/10.1172/jici.

insight.133817.

\section{Introduction}

A characteristic feature of chronic kidney disease (CKD) is the retention and accumulation of protein-bound and other small molecules (uremic solutes) that circulate in the plasma of the patient (1). While potentially functioning as toxins, many of these small-molecule compounds possess signaling capabilities; as their plasma concentration increases in the setting of progressive kidney failure, their potential to affect metabolism and signaling increases as well. However, the actual alterations in metabolic pathways in the setting of CKD are only beginning to be worked out. To make further progress, it is important to define how these molecules are handled in the setting of CKD.

Recently, there has been renewed interest in the relative importance of renal tubular secretory capacity in the setting of declining renal function in the context of the handling of drugs and organic solutes, including uremic solutes and uremic toxins (2-4). Growing evidence suggests the residual renal secretory capacity - especially via the organic anion transport (OAT) system — may be particularly important in the setting of CKD (2-8). In fact, it is believed that residual function of the proximal tubule is likely to be central to the removal of compounds not generally cleared by hemodialysis, which include many of the protein-bound small-molecule uremic solutes and toxins (2, 3, 7, 9-13).

Many of these protein-bound uremic solutes/toxins and other endogenous metabolites accumulating in renal insufficiency, as well as many of the drugs (e.g., antibiotics, antivirals) administered to patients with $\mathrm{CKD}$, are, at physiological $\mathrm{pH}$, organic anions. In normal physiological settings, the renal uptake and elimination of such small-molecule organic anions is mediated by the OAT system of the kidney, in particular the major renal drug and toxin transporters, OAT1 (also known as SLC22A6, originally identified as NKT) and OAT3 (also known as SLC22A8) $(10,14)$. OAT1 and OAT3 are expressed on the basolateral membrane to the proximal tubule, where they mediate the uptake of small-molecule organic anion com- 
pounds from the blood and into the cell. Such compounds include a wide array of commonly prescribed drugs, endogenous metabolites, signaling molecules, and toxins (15-24).

In the case of uremic toxins, basolateral influx via the OATs on the blood side may enable efficient elimination via the apical efflux pathway - mediated by MRPs (ABCC2 and ABCC3) and other transporters - if tubular secretion is intact, allowing elimination of the uremic toxins into the lumen (3). However, if the luminal efflux pathway is not sufficiently active, then potential proximal tubule cell toxins, such as indoxyl sulfate, can, once taken up by OAT1 and OAT3, accumulate in the cell; this cell damage is, at least in part, thought to lead to progressive renal disease $(25,26)$. Thus, tubular OAT function in the setting of renal insufficiency is, potentially, a double-edged sword; it is critical for the elimination of uremic solutes and toxins, but transport of molecules such as urate and indoxyl sulfate into damaged or injured proximal tubule cells may lead to further tubular damage (27). Hence, it is essential to understand the OAT-transported metabolites that accumulate in the setting of renal insufficiency. Furthermore, understanding the OAT-dependent metabolic pathways may set the stage for modulating the aberrant metabolism that occurs as renal function declines, potentially by altering OAT1 or OAT3 expression and/or function (28-30).

Here, we considered renal insufficiency as a complex metabolic disease. In particular, we evaluated the role of OAT1 and OAT3 in this process and, through the lens of the remote sensing and signaling theory, in the remote interorgan and interorganismal (gut-microbiome-host) flow of metabolites and signaling molecules that is disrupted in $\operatorname{CKD}(14,17,18,23,24,31)$. Using the subtotal nephrectomy (STN) model for CKD (32), we identified those metabolites accumulating in the setting of substantially decreased glomerular filtration rates (GFR) and decreased tubular secretion of para-amino hippurate (PAH), the prototypical organic anion substrate. Metabolomics analyses were performed on the plasma of adult rats 2 weeks after STN and compared with those of sham-operated animals. Moreover, in order to investigate the role of tubular secretion in the setting of renal insufficiency, STN animals were also treated with and without the OAT inhibitor, probenecid (2-hour short-term treatment). The sets of metabolites handled by the OAT system, which represent a measure of residual tubular secretion of anions via the proximal tubule, were then identified. Focusing on uremic solutes and toxins, metabolomics data from probenecid-treated animals were then analyzed in the context of metabolomics data obtained from Oat1-KO and Oat3-KO mice (15, 20-22, 33); we also analyzed human and rodent metabolomics data for the contribution of the gut microbiota to the uremic solute profile. These comparisons helped us to define the key metabolic pathways dependent upon OAT-mediated remote interorganismal (gut microbe-host) communication in the setting of CKD.

\section{Results}

STN affects glomerular filtration and tubular secretion. Whole kidney glomerular filtration rates (GFR) were significantly lower in STN rats $(2.5 \pm 0.4 \mathrm{ml} / \mathrm{min})$ compared with those in sham-treated controls $(4.8 \pm$ $0.4 \mathrm{ml} / \mathrm{min}, P \leq 0.05$ ), reflecting the effects of reduced nephron mass (Figure 1 ). The $>80 \%$ reduction in renal mass ( 5 of 6 nephrectomy) also led to a significant reduction in the clearance of para-aminohippuric acid $(\mathrm{PAH})$ - the prototypical organic anion transported in vivo by OAT1 and, to a lesser degree, by OAT3 $(15,34,35)$ - such that its clearance was similarly reduced by half (from a high of roughly $12 \mathrm{ml} / \mathrm{min}$ in sham-operated animals to around $6 \mathrm{ml} / \mathrm{min}$ in the STN animals) (Figure 1).

Uremic solutes in the plasma of animals with renal insufficiency. In order to clarify the effects of these reductions in GFR and tubular secretion on uremic solutes and other metabolites, metabolomics analyses were performed on plasma collected from both sham-operated controls and STN animals. Partial least squares discriminant analysis revealed a clear separation of the different treatment groups based on the profile of the 668 metabolites of known identity that were detected on the platform (Figure 2). Almost $50 \%$ of the metabolites (326 of 668) displayed significantly $(P \leq 0.05)$ altered (increased or decreased) plasma concentrations in the setting of progressive renal insufficiency (with 315 showing increased plasma concentrations and 11 displaying reductions in plasma concentration) (Figure 2). Moreover, metabolite set enrichment analysis further revealed that each of the 8 superpathway metabolic categories described in the metabolomics analyses (see Methods) contained at least 1 of the altered metabolites, indicating the broad effects of STN on systemic metabolism (Supplemental Figure 1; supplemental material available online with this article; https://doi.org/10.1172/jci.insight.133817DS1).

Since a characteristic of a so-called "uremic metabolism," such as that seen here, is the increased presence of uremic retention solutes/uremic toxins in the plasma due to reduced GFR, as well as reduced uptake and elimination via the kidney proximal tubule (i.e., tubular secretion) $(31,36,37)$, we decided to 
A

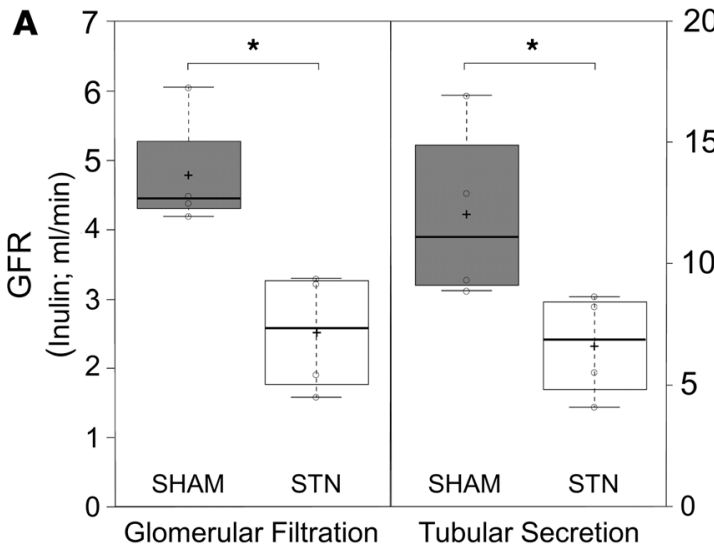

20

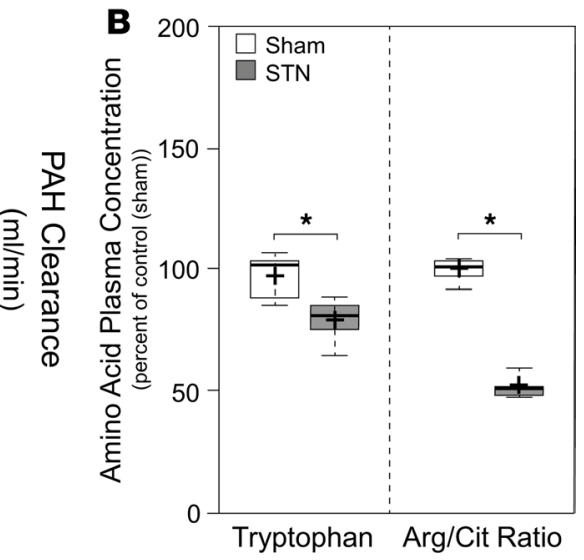

Figure 1. Subtotal nephrectomy alters kidney function, leading to differences in plasma metabolite profiles. (A) Box-and-whisker plots illustrating the effects of subtotal nephrectomy on the glomerular filtration rate (GFR; left side of graph), as measured by $\mathrm{ml} / \mathrm{min}$ of inulin clearance, and tubular secretion (right side), as measured by the clearance of para-aminohippurate (PAH). Two weeks after STN surgery there was a significant reduction in both GFR $\left(\sim 50 \%\right.$; $\left.{ }^{*} \leq \leq 0.05\right)$ and tubular secretion $\left(\sim 50 \% ;{ }^{*} P \leq 0.05\right)$ in the STN animals (white rectangles) compared with the sham-operated animals (gray rectangles). Box plots were generated using the functionalities of BoxPlotR (92) (5 animals were used for each experimental group; a 2-tailed Student's $t$ test was used to determine statistical significance; a $P$ value less than 0.05 was considered significant). (B) Box-and-whisker plots of the changes in amino acid concentrations following STN. The plasma concentration of tryptophan was significantly reduced following STN. Additionally, there were significant reductions in the plasma ratios of arginine to citrulline ${ }^{*} P \leq 0.05$ ). Black cross in each box represent the mean; solid black center lines indicate the median; open circles represent the individual data points; box limits indicate the 25 th and 75 th percentiles as determined by $\mathrm{R}$ software; whiskers extend 1.5 times the interquartile range from the 25 th and 75 th percentiles; outliers are represented by dots (92) (5 measures of plasma concentration were used for each experimental group; a 2-tailed Student's $t$ test was used to determine statistical significance; a $P$ value less than 0.05 was considered significant).

focus our attention on these types of metabolites as a way to narrow the focus of the analysis. With this in mind, a list of 293 uremic solutes identified by a number of expert groups was compiled (Supplemental Table 1), of which 232 were among the 668 identifiable compounds on the metabolomics platform (Figure 2 and Supplemental Table 1). In the analysis, which was comprised of both uremic solutes that had significantly $(P \leq 0.05)$ accumulated in the plasma of the STN animals as well as those approaching significance $(0.05<P<0.10)), 165$, or approximately $71 \%$, were observed to accumulate in the plasma of the STN rats (Figure 2 and Supplemental Table 1). This list included a number of well-known retention solutes (e.g., urea, indoxyl sulfate, p-cresol sulfate, TMAO, methylguanidine, and others) (Figure 2 and Supplemental Table 1). Examination of this subset of metabolites revealed that uremic solutes with increased plasma concentration were present in all of the metabolic superpathways (data not shown).

Probenecid blocks PAH secretion in STN. Given the focus on the contribution of tubular secretion of organic anions in uremic metabolism seen in the setting of renal insufficiency, the STN animals were exposed to the well-established inhibitor of the proximal tubule OAT system, probenecid. This transport system, an important component of tubular secretion, is involved in the uptake and elimination of a substantial number of protein-bound organic anion retention solutes that are not readily filtered by the glomerulus (3).

To confirm the inhibitory effect of probenecid on the tubular secretion of organic anions, the clearance of PAH was determined in sham-treated rats versus STN animals in the absence and presence of probenecid. Probenecid-sensitive PAH clearance is operationally considered to be a reasonable measure of OAT-mediated transport (38-40). As expected, treatment with a single $200 \mathrm{mg} / \mathrm{kg}$ dose of probenecid revealed little to no effect on GFR (as measured by inulin clearance) in either the sham-treated controls or STN animals (Figure 3); however, OAT-mediated tubular secretion of organic anions (as measured by the clearance of PAH) was significantly reduced by probenecid treatment in both the sham-operated controls and in the STN animals (Figure 3). For example, PAH clearance in the sham-operated control animals was reduced from $12 \mathrm{ml} / \mathrm{min}$ to approximately $6 \mathrm{ml} / \mathrm{min}$ following the administration of probenecid. Moreover, treatment of the STN animals with probenecid resulted in an additional 50\% reduction in PAH clearance, such that PAH clearance in the STN probenecid-treated animals was reduced from a high of $12 \mathrm{ml} / \mathrm{min}$ in the control sham-treated animals to a low of approximately $3 \mathrm{ml} / \mathrm{min}$ (an overall 4 -fold reduction in PAH clearance) - indicating a 
A

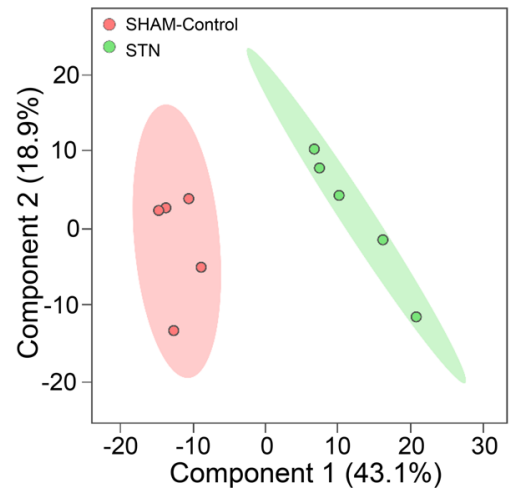

B

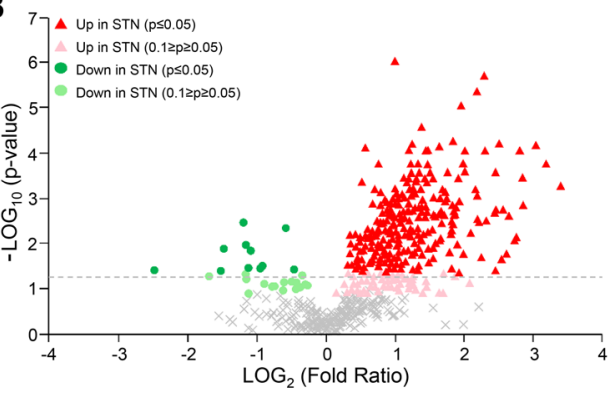

C $77 \Delta$ Up in $\operatorname{STN}(p \leq 0.05)$

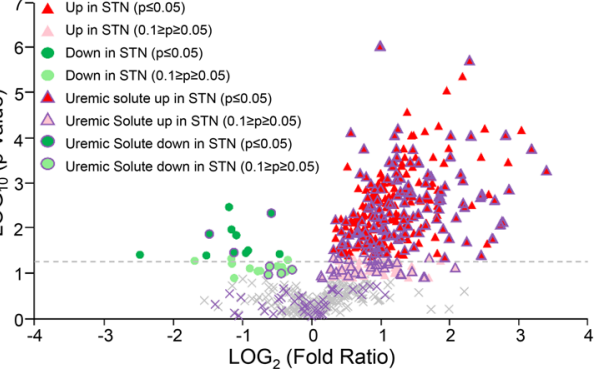

D

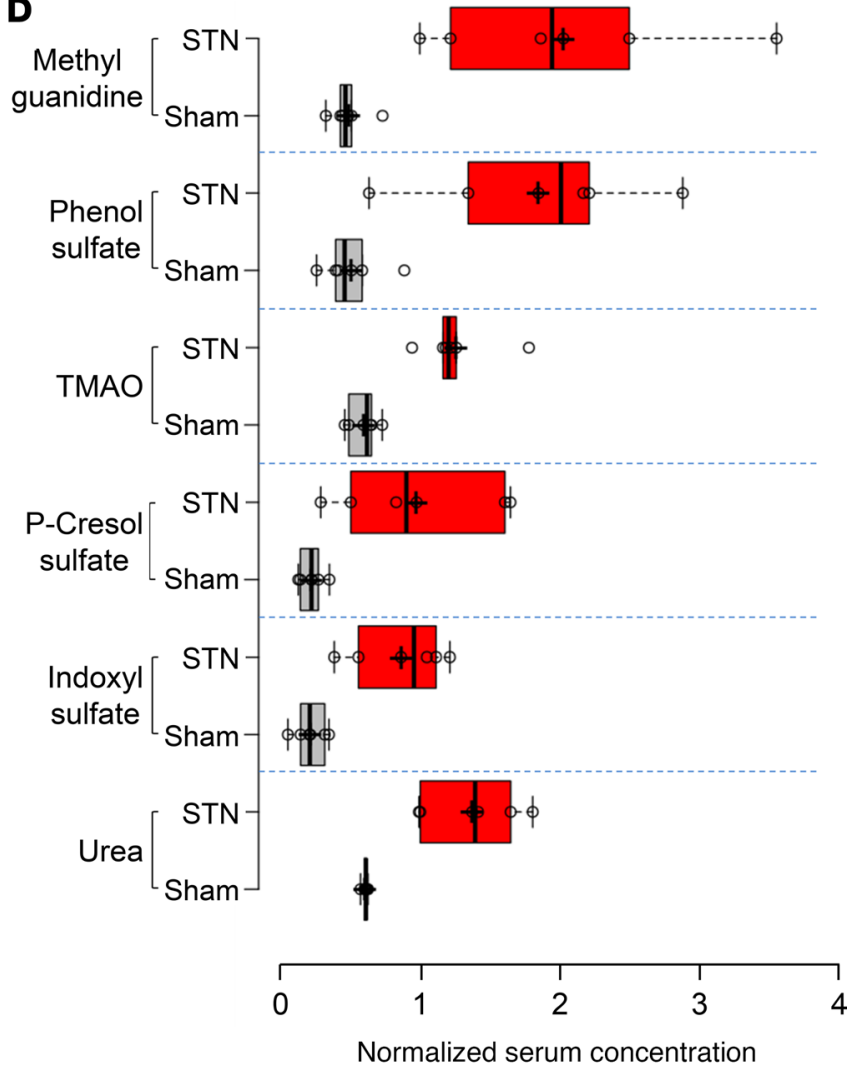

Figure 2. Analysis of uremic solutes found on the metabolomics platform. (A) Partial least squares discrimination analysis of the metabolomic profiling data reveals clear separations between serum/plasma metabolites obtained from sham-operated animals (control; red) and subtotal nephrectomized animals (STN; green). (B and C) Volcano plots displaying metabolomics data derived from the analysis of the serum obtained from sham-operated control and STN animals. The negative logarithm of the $P$ value for each metabolite is plotted against the logarithm to the base 2 of the fold change (sham treated vs. STN). Each point in the plot represents an individual metabolite. (B) The red triangles indicate those metabolites accumulating in the serum of the STN animal either significantly $(P \leq 0.05)$ or with a trend towards significance $(0.1 \geq P \geq 0.05)$, the green dots represent those metabolites decreased in the serum of the STN animal $(P \leq 0.05$ or $0.1 \geq p \geq 0.05$ ), while the gray crosses indicate those metabolites not significantly altered in the STN animal. (C) The same image as B except that the 165 suspected uremic solutes/toxins found on the metabolomics platform are highlighted in purple. The majority of these metabolites are accumulating in the serum of the STN animal. (D) Box-and-whisker plots of the changes in concentrations in sham-treated controls (gray boxes) versus STN animals (red boxes) for some representative uremic toxins/solutes (5 measures of plasma concentration were used for each experimental group; a 2-tailed Student's $t$ test was used to determine statistical significance; a $P$ value less than 0.05 was considered significant).

clear and dramatic effect of probenecid on OAT-mediated tubular secretion in the STN animals (Figure 3). Thus, roughly half of the measurable PAH clearance, in either the sham-treated or STN animal, was inhibited by a 2-hour exposure to probenecid (Figure 3) - operationally supporting substantial involvement of 1 or more OATs in tubular organic anion handling in the setting of renal insufficiency in the STN model. Therefore, probenecid-sensitive increases in the plasma levels of uremic solutes were examined in order to provide insight into the role of tubular secretion in the setting of renal insufficiency.

Identification of 58 probenecid-sensitive uremic solutes/toxins in STN. As with the comparison between the sham-operated controls and the STN animals, partial least squares discriminant analysis indicated a clear separation of the short-term treated probenecid STN group from the STN group (Figure 3). Since we were interested in the contribution of tubular secretion to the uptake of uremic toxins/solutes, we focused our attention on those uremic retention solutes that accumulated in the serum of the STN rat following exposure to probenecid. This analysis revealed that, of the 232 uremic solutes present as identifiable compounds on the metabolomics platform (Supplemental Table 1), one-quarter of them (i.e., 58 of 232) were increased 
A

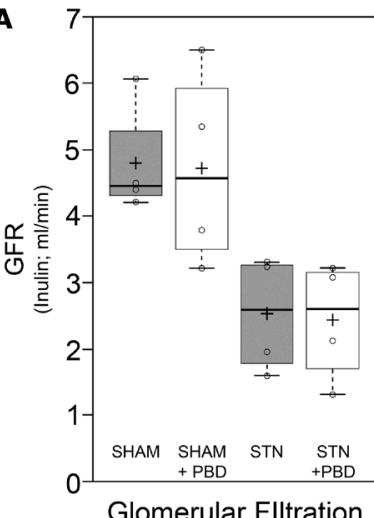

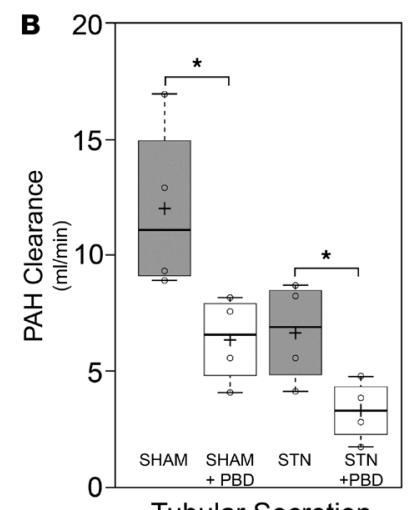

Tubular Secretion

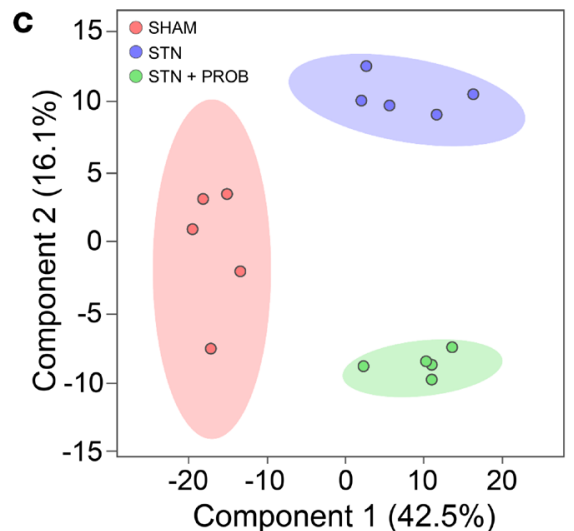

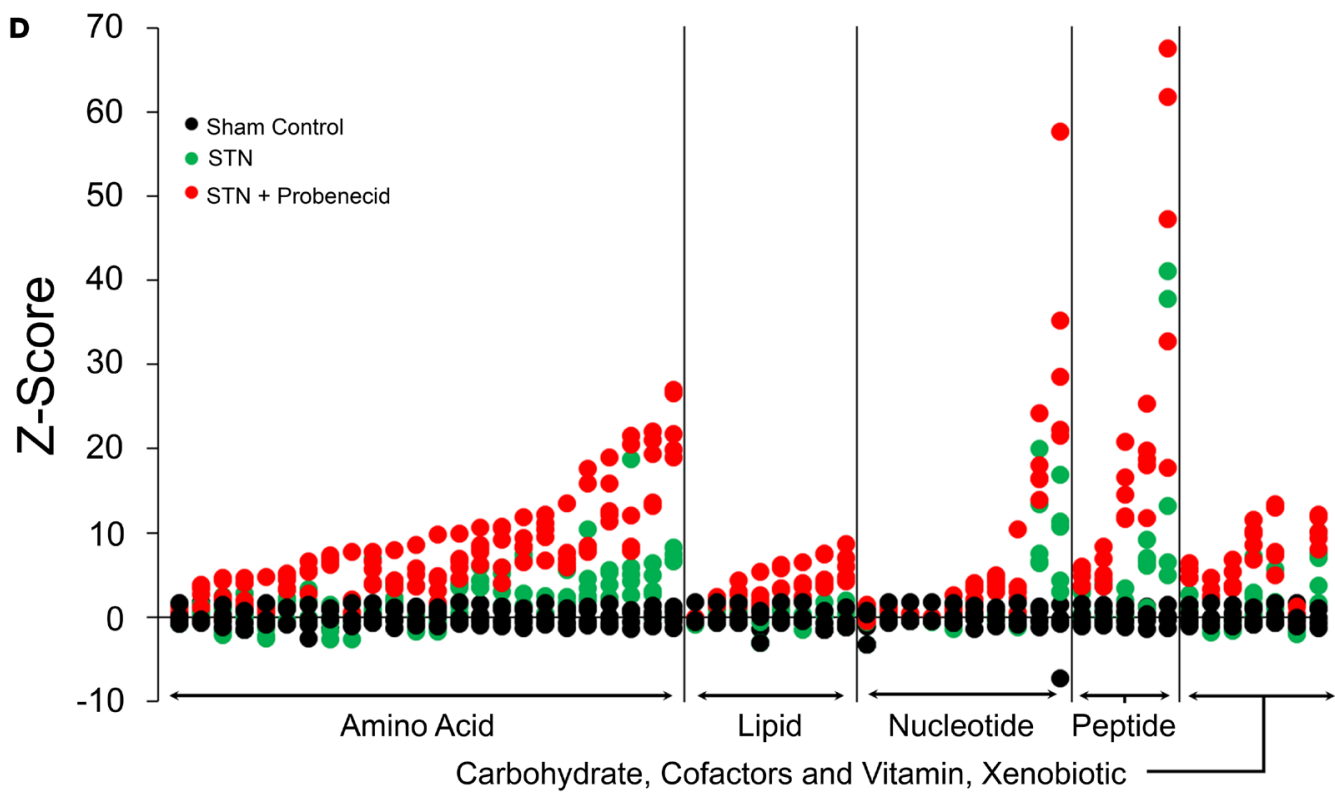

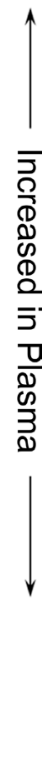

\section{Metabolic Superpathway}

Figure 3. Probenecid treatment affects tubular secretion but not glomerular filtration, leading to clear differences in metabolite profiles of uremic solutes. (A and B) Box-and-whisker plots illustrating the effects of probenecid treatment on GFR (A) and tubular secretion (B). After treating the animals with probenecid, there was little to no effect on GFR in either the sham-operated animals or STN animals (A). Probenecid (PBD) treatment, however, had a significant ( ${ }^{*} P$ $\leq 0.05$ ) effect on tubular secretion (B), such that, in both the sham-operated and the STN animals after probenecid treatment, there was a dramatic reduction in PAH clearance (PBD, probenecid; gray boxes, untreated sham-operated controls and untreated STN animals; white boxes, probenecid-treated sham-operated controls and STN animals). As in Figure 1, black cross in each box represents the mean; solid black center lines indicate the median; open circles represent the individual data points; box limits indicate the 25 th and 75 th percentiles, as determined by $R$ software; whiskers extend 1.5 times the interquartile range from the 25th and 75th percentiles, outliers are represented by dots (92) (5 animals were used for each of the experimental groups; a 2-tailed Student $t$ test was used to determine statistical significance; a $P$ value less than 0.05 was considered significant.) (C) Partial least squares discrimination analysis of the metabolomic profiling data reveals clear separations between serum/plasma metabolites obtained from untreated sham-operated animals (control; red), untreated STN animals (STN; purple), and probenecid-treated STN animals (STN+PROB; green). (D) A Z score plot of metabolite intensities for the 58 uremic solutes affected by treatment of the STN animals with probenecid. The greater the distance of each dot (individual measurements for each metabolite) from the $x$ axis, the greater the magnitude of change in plasma concentration for each metabolite (black circles, sham-treated control; green circles, STN rats; red circles, STN rats treated with probenecid). In this case, the distance of the green dot (STN alone) from the $x$ axis is the average score of the STN animal above the sham-treated control (black dot), while the distance of the red dot (STN with probenecid) from the $x$ axis is the average score of the probenecid-treated STN animal above the untreated STN animal. Plasma increases in metabolite concentration (positive $Z$ scores) are seen above the $x$ axis, while decreases in plasma concentration are represented by those dots below the $x$ axis. As can be seen, many metabolites belonging to the amino acid metabolic superpathway show significant accumulation in the plasma of the STN animal following probenecid treatment.

in the plasma of the probenecid-treated STN animal compared with that of untreated STN animals (both $P \leq 0.05$ and $0.05<P<0.10$ ) (Table 1).

In order to discriminate the metabolite alterations induced by treatment with probenecid, a $Z$ score plot was used to compare the intensities of the samples altered in the serum of the probenecid-treated STN animal relative to the intensities of the samples in the sham-treated control and in the STN ani- 
mal (Figure 3). The $Z$ score, also called the standard score or normal score, is a dimensionless quantity derived by subtracting the control population mean from an individual raw score (which centers the distribution) and then dividing the difference by the control population standard deviation (which standardizes the distribution). The $Z$ score indicates how many standard deviations an observation is above or below the mean of the control group. $Z$ scores are used to compare observations coming from different distributions. From this plot, in which each dot represents an individual measurement, it is evident that probenecid treatment leads to significant increases in the serum concentration of these 58 metabolites in the STN animal (Figure 3). Among the 58 uremic retention solutes displaying significant or nearly significant increases in the plasma of the probenecid-treated STN animals, $23(\sim 40 \%)$ of these metabolites were either decreased or not significantly (nor nearly significantly) increased in the plasma of the untreated STN animal. The data strongly suggest that these 58 uremic solutes/toxins are likely handled by the organic anion secretory pathway of the proximal tubule, which represents an important residual function of the proximal tubule in the setting of renal insufficiency.

Probenecid-sensitive metabolic pathways in STN. Analysis of these uremic retention solutes revealed that, although probenecid-sensitive metabolites were found to be components of several metabolic "superpathways," the majority of them were found to be components of the amino acid superpathway (Table 1 and Figure 4). Examination of the amino acid superpathway revealed that the tryptophan metabolism "subpathway" contained the most probenecid-sensitive metabolites (Table 2). Pathway enrichment analysis, which not only takes into account the number of uremic solutes increased in the plasma of the probenecid-treated STN animal, but it also puts these metabolites in the context of all amino acid superpathway-associated metabolites analyzed, as well as all uremic solutes affected by probenecid treatment, was performed. In this case, similar to what was seen above, there was a dramatic enrichment in metabolites associated with tryptophan metabolism (Table 2), although one could also see that there was a similar enrichment for several other amino acid metabolism subpathways (Figure 4).

While the actual probenecid-sensitive solutes are varied, the fact that the plasma concentration of some metabolites was found to be markedly increased in STN animals exposed to probenecid, including those not increased in the STN condition alone, raises the possibility that, at some level, probenecid-mediated inhibition of the OAT component, shown to be largely mediated by the OATs, captures a key identifying aspect of declining residual tubular secretion. For example, the progression of CKD in humans has been characterized by measurable changes in the plasma levels and ratios of certain amino acids (41). For instance, tryptophan levels are lower in the plasma of stage 4-5 CKD compared with earlier stage 2-3 CKD (41). In the metabolomics analyses performed here, reduced levels of tryptophan were not only seen in the plasma of the STN animal compared with the sham-treated control (Figure 1), but the plasma level of this essential amino acid was further reduced following probenecid treatment of the STN animal compared with the untreated STN animal (albeit this did not reach statistical significance). Furthermore, reductions in the ratios of tyrosine to phenylalanine and arginine to citrulline - also potential markers of worsening CKD (42) - were seen in the probenecid-treated STN animals compared with the untreated animals. Late-stage CKD has also been correlated with increases in the ratio of ornithine to arginine (42), which were also seen in the probenecid-treated STN animals. Although these changes in amino acid concentration in CKD have been attributed to many factors, including alterations in protein metabolism/catabolism as well as reduced protein intake as a result of malnutrition in patients with $\mathrm{CKD}$, the data described here - which show that a short-term treatment of the STN animal with probenecid leads to alterations in several biomarkers that are reminiscent of progressing $\mathrm{CKD}$ - suggest that these changes are, at least in part, due to decreases in OAT-mediated tubular secretion and the reduced clearance of these metabolites from the plasma.

Comparison of STN-probenecid data to that from Oat1-KO and Oat3-KO animals. Since probenecid treatment inhibits OAT-mediated uptake from the blood and into the proximal tubule, we further analyzed the uremic solutes by comparing them to metabolites that accumulate in the plasma of the Oat1-KO and the Oat3-KO animals $(15,16,20-22,33,43-45)$. The metabolites common between the metabolomics platforms used in the various analyses were overlapped, and a set of uremic solutes were identified that were probenecid sensitive in the STN animal and also increased in the plasma of the Oat-KO animals (Table 1). Of these probenecid-sensitive metabolites, the majority of them displayed increased plasma concentrations in either the Oat1-KO animal alone or in both the Oat3-KO and Oat1-KO animals, while several were found to accumulate in the plasma of the Oat3-KO animal but not the Oat1-KO animal (Table 1). In addition, roughly one-quarter of these metabolites with increased plasma concentration in the probenecid-treated STN ani- 
Table 1. Uremic solutes/toxins increased in plasma of probenecid-treated STN animal

\begin{tabular}{|c|c|c|c|c|c|}
\hline \multirow[t]{12}{*}{ Amino acid } & Alanine and aspartate metabolism & $\mathrm{N}$-methylalanine & & & \\
\hline & Glutamate metabolism & $\alpha$-Ketoglutaramate & $x$ & & \\
\hline & Leucine, isoleucine and valine metabolism & $\begin{array}{l}\text { Isobutyrylglycine } \\
\text { Isobutyrylcarnitine }\end{array}$ & $\begin{array}{l}x \\
x\end{array}$ & & \\
\hline & $\begin{array}{l}\text { Methionine, cysteine, SAM and taurine } \\
\text { metabolism }\end{array}$ & $\begin{array}{l}\text { Cystine } \\
\mathrm{N} \text {-formylmethionine } \\
\text { Hypotaurine } \\
\text { Cysteine }\end{array}$ & $\begin{array}{l}x \\
x \\
x\end{array}$ & $\begin{array}{l}x \\
x\end{array}$ & \\
\hline & Phenylalanine metabolism & $\mathrm{N}$-acetylphenylalanine & & & \\
\hline & Polyamine metabolism & $\begin{array}{l}\text { Putrescine } \\
\text { 5-Methylthioadenosine } \\
\text { 4-Acetamidobutanoate }\end{array}$ & $\begin{array}{l}X \\
X\end{array}$ & $\begin{array}{l}x \\
x\end{array}$ & $x$ \\
\hline & Tryptophan metabolism & $\begin{array}{c}\text { Indolelactate } \\
\text { 3-Indoxyl sulfate } \\
\text { Indole-3-carboxylic acid }\end{array}$ & & $\begin{array}{l}x \\
x\end{array}$ & $\begin{array}{l}X \\
X \\
X\end{array}$ \\
\hline & & Xanthurenate & $x$ & $x$ & \\
\hline & & Kynurenate & $X$ & & \\
\hline & & Kynurenine & $x$ & & \\
\hline & & $\begin{array}{c}\mathrm{N} \text {-formylanthranilic acid } \\
\text { Anthranilate }\end{array}$ & & & $x$ \\
\hline & Tyrosine metabolism & 3-(4-Hydroxyphenyl) lactate & $x$ & $x$ & $x$ \\
\hline Carbohydrate & Aminosugar metabolism & Glucuronate & $x$ & $x$ & $\mathrm{x}$ \\
\hline $\begin{array}{l}\text { Cofactors and } \\
\text { vitamins }\end{array}$ & Ascorbate and aldarate metabolism & $\begin{array}{l}\text { Threonate } \\
\text { Oxalate (ethanedioate) } \\
\text { Gulonate }\end{array}$ & $\begin{array}{l}x \\
x\end{array}$ & & \\
\hline Lipid & Fatty acid metabolism (acyl carnitine) & $\begin{array}{l}\text { Laurylcarnitine (C12) } \\
\text { Octanoylcarnitine (C8) }\end{array}$ & $x$ & & \\
\hline Nucleotide & Pyrimidine metabolism & $\begin{array}{c}\text { Cytidine } \\
\text { Orotate } \\
\text { Thymidine } \\
\text { Thymine } \\
\mathrm{N} \text {-acetyl- } \beta \text {-alanine } \\
\text { 3-Ureidopropionate } \\
\text { Uracil }\end{array}$ & $\begin{array}{l}x \\
x\end{array}$ & $x$ & $x$ \\
\hline \multirow[t]{2}{*}{ Peptide } & Acetylated peptides & Phenylacetylglycine & $x$ & & $x$ \\
\hline & $\gamma$-Glutamyl amino acid & $\begin{array}{c}\gamma \text {-Glutamylthreonine } \\
\gamma \text {-Glutamylisoleucine } \\
\gamma \text {-Glutamylphenylalanine } \\
\gamma \text {-Glutamylvaline }\end{array}$ & $\begin{array}{l}X \\
X \\
X\end{array}$ & & $x$ \\
\hline \multirow[t]{2}{*}{ Xenobiotics } & Chemical & $\begin{array}{c}\text { S-(3-hydroxypropyl) mercapturic acid } \\
\text { 6-Hydroxyindole sulfate } \\
\text { Thioproline } \\
\text { 3-Hydroxyindolin-2-one sulfate }\end{array}$ & $\begin{array}{l}X \\
X \\
X\end{array}$ & $\begin{array}{l}X \\
X \\
X\end{array}$ & $\begin{array}{l}X \\
X \\
X\end{array}$ \\
\hline & Drug & Hydroquinone sulfate & $x$ & $x$ & \\
\hline
\end{tabular}

Probenecid-sensitive uremic solutes found on the Metabolon platform. Column 1 indicates the metabolic super pathway to which each metabolite belongs. Column 2 indicates the metabolic subpathway, and column 3 indicates each individual uremic solute/toxin found on the metabolomic platform with increased plasma concentration in the setting of CKD. A mark in column 4 (Oat1-KO) indicates that this metabolite was also accumulated in the plasma of the Oat1-KO animal. A mark in column 5 (Oat3-KO) indicates that the uremic solute/toxin also displayed plasma accumulation in the Oat3-KO animal. The absence of a check mark in columns 4 or 5 indicates that no significant plasma accumulation was detected for that metabolite in either the Oat7-KO or Oat3-KO animal. A mark in column 6 indicates that the uremic solute was found to be colon derived in other metabolomics analyses $(47-50,53-62,78,93,95)$. 
Table 2. Probenecid-sensitive uremic solutes/toxins

\begin{tabular}{|c|c|c|c|c|c|}
\hline \multirow[t]{2}{*}{ Superpathway } & \multirow[t]{2}{*}{ Subpathway } & \multicolumn{2}{|c|}{ Increased in plasma } & \multirow[t]{2}{*}{ Total no. } & \multirow[t]{2}{*}{ Enrichment } \\
\hline & & STN & STN-PBD & & \\
\hline \multirow[t]{9}{*}{ Amino acid } & Tyrosine metabolism & 5 & 1 & 9 & 0.49 \\
\hline & Alanine and aspartate metabolism count & 4 & 1 & 5 & 0.88 \\
\hline & Leucine, isoleucine, and valine metabolism & 5 & 2 & 10 & 0.88 \\
\hline & Glutamate metabolism & 4 & 1 & 4 & 1.10 \\
\hline & Methionine, cysteine, SAM, and taurine metabolism & 7 & 4 & 10 & 1.77 \\
\hline & Glutathione metabolism & 2 & 1 & 2 & 2.21 \\
\hline & Phenylalanine metabolism & 1 & 1 & 2 & 2.21 \\
\hline & Polyamine metabolism & 4 & 3 & 6 & 2.21 \\
\hline & Tryptophan metabolism & 10 & 10 & 13 & 3.40 \\
\hline
\end{tabular}

Pathway enrichment analysis (see Methods) of uremic solutes involved in the amino acid superpathway accumulating in the serum of the probenecidtreated STN animal (STN-PBD). The most affected metabolic pathways are shown and are ranked by their enrichment. According to Metabolon ( 94$)$, a value greater than 1 indicates that a pathway contains more statistically altered compounds relative to the overall study. In this case, the compounds were limited to the uremic solutes/toxins found on the platform (Supplemental Table 1). Total no. (column 5) refers to the number of uremic solutes/toxins associated with each metabolic subpathway that are detectable on the metabolomics platform. For example, in the subpathway labeled "Tryptophan metabolism," of the total of 13 uremic solutes/toxins represented on the metabolomics platform and associated with this pathway, 10 were elevated in the STN versus sham comparison and 10 were elevated in the STN-PBD versus STN comparison. However, this does not necessarily imply that metabolites elevated in the STN-PBD versus STN comparison are the same ones that are elevated in the STN versus sham comparison. In other words, these may be different subsets of the 13 .

mals, which were also increased in the plasma of the Oat $1-\mathrm{KO}$, the Oat3-KO, or both $\mathrm{KO}$ animals, were found to be components of tryptophan metabolism (Figure 4).

Since these uremic toxins were (a) probenecid sensitive and (b) increased in the plasma of one or both of the OAT-KO animals, these data strongly suggest that these metabolites not only interact with the OATs but are more than likely to be substrates of these proximal tubule transporters. Moreover, since the majority of these metabolites was increased in the plasma of the Oat1-KO animal (Table 1), this also raises the possibility that, at least in vivo and at the concentrations used in this study, probenecid has a substantially greater inhibitory effect on OAT1-mediated transport than it does on that mediated by OAT3. This is consistent with the $\mathrm{KO}$ data indicating that at least $75 \%$ of PAH transport is mediated by OAT1 (15).

Comparison to colon-derived endogenous metabolites. It is increasingly appreciated that many uremic solutes are derived from the colon, either through the action of colonic microbes or from colon cell metabolism (46). In the metabolomics analyses performed here, presumptive colon-derived uremic toxins were identified on the metabolomics platform (47-62) (Table 1). Roughly 75\% of these were found to accumulate in the plasma of the STN rat, while 18 displayed increased plasma concentration in the probenecid-treated STN animal (Table 1). These metabolomics data were then overlapped with data from the Oat1-KO and Oat3-KO animals, and probenecid-sensitive colon-derived uremic solutes were also found to be increased in the plasma of the Oat-KO animals - indicating interaction with the organic anion transporters (Table 1). Thus, this remote interorganismal communication connects metabolites produced by colonic microbes in the setting of CKD to blood-urine barrier via OAT1 and OAT3.

\section{Discussion}

There has recently been a renewed interest in understanding the secretory activity remaining in the proximal tubules of the failing kidney $(2-4,6)$. This so-called residual renal function has been previously largely viewed as fluids and solutes derived from the glomerular filtrate; however, recent data indicates that residual renal function should also include tubular secretion involved in the elimination of solutes that are not eliminated via glomerular filtration, particularly those solutes considered to be uremic solutes/toxins $(2-4,6)$.

Since many of the uremic solutes are small-molecule organic anions, a substantial fraction of residual tubular secretion is likely to be modulated/regulated by the OAT system of the kidney. The rate-limiting step of this transport system of the kidney is mediated by OAT1 (63) and OAT3, organic anion transporters found on the basolateral surface of proximal tubule cells where they function in the uptake and clearance of small-molecule organic anions from the blood and into the cell $(14,17,18)$. These transporters have been 
A

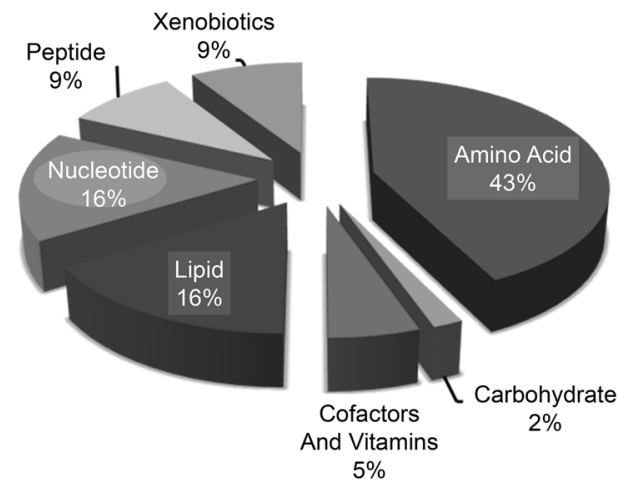

B

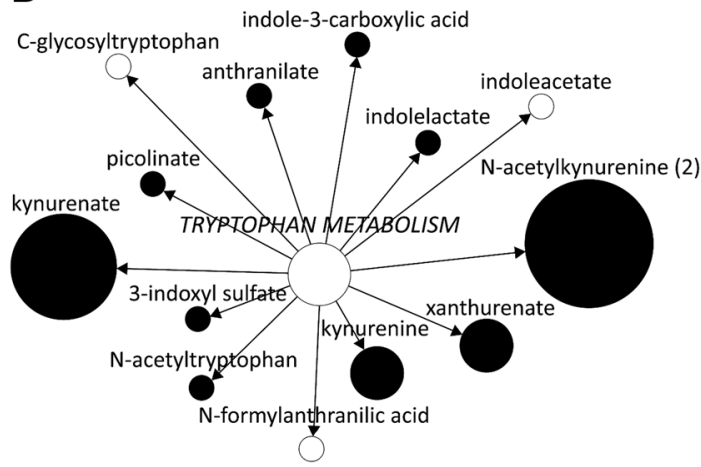

C

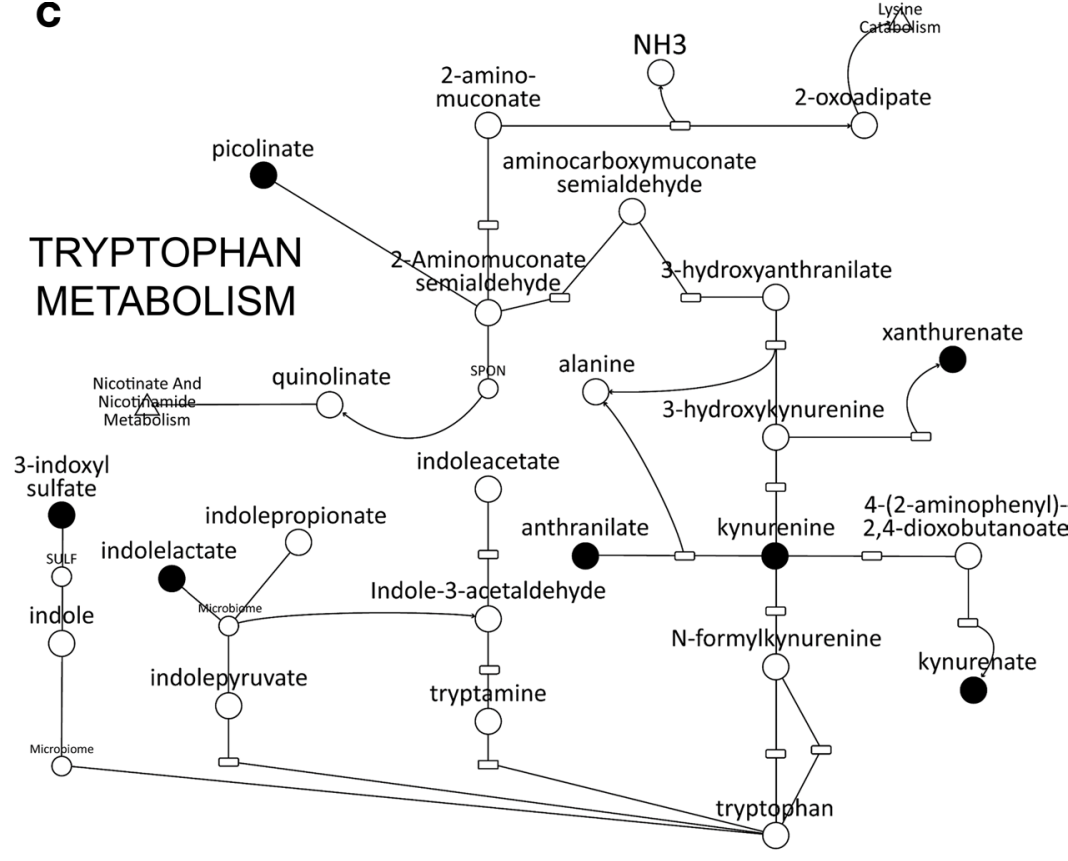

Figure 4. Overlap of uremic solutes found on metabolomics platform with those increasing in the plasma of the STN animal in the presence of probenecid. (A) Pie chart representing the percentage of increased metabolites within each superpathway of metabolism, specifically amino acids, lipids, xenobiotics, peptides, cofactors and vitamins, nucleotides, and carbohydrates. (B) Probenecid-sensitive uremic solutes associated with tryptophan metabolism. The white circles indicate those metabolites that did not display increases in their plasma concentration, and the black circles indicate metabolites for which increases in plasma concentration were found. The size of the black circle is based upon the magnitude of the change in plasma concentration (i.e., the larger the circle the greater the accumulation of that metabolite in the plasma of the probenecid-treated STN animal). (C) Representation of the tryptophan metabolism pathway. The black dots represent uremic solutes that are depicted in this representation of the tryptophan metabolic pathway and which were found to probenecid sensitive in the STN animal.

shown to mediate the uptake and elimination of a wide range of organic anions, including drugs, endogenous metabolites, signaling molecules, antioxidants, and toxins (including uremic toxins) $(14,17,18)$. In $\mathrm{CKD}$, the expression and function of these transporters has been shown to be altered (64-66).

Using STN in rats as a model of chronic renal failure, we investigated the contribution of the OAT system to residual renal function, with a particular emphasis on the uremic solutes and uremic toxins. These included well-characterized small molecules, such as indoxyl sulfate, kynurenate, anthranilate, and xanthurenate. Importantly, within the set of 46 probenecid-sensitive uremic solutes/toxins, 14 did not display significant accumulation in the plasma of the untreated STN animal (Figure 3) but were found to significantly accumulate in the plasma of the probenecid-treated STN animal (Table 1). These uremic solutes included such substances as kynurenine, putrescine, oxalate, and N2,N2-dimethylguanosine (Table 1).

While the actual probenecid-sensitive solutes are involved in a variety of metabolic pathways, the fact that their plasma concentration was significantly increased in the presence of probenecid (but not in STN alone) suggests potential clinical relevance. For example, these uremic solutes might represent markers of late-stage $\mathrm{CKD}$. Since progressive $\mathrm{CKD}$ is characterized by declining renal function, including a continuous loss of residual proximal tubule function - of which OAT-mediated uptake of organic anions from the blood is an important component - one can hypothesize that treatment with probenecid is, at least at some level, mimicking late-stage renal insufficiency, with severely reduced OAT-mediated clearance. Thus, the significant plasma accumulation of some metabolites following treatment with probenecid raises the possibility that these compounds could represent biomarkers of declining residual secretory function in CKD. This could be important clinically, as this could potentially help in determining how much residual function remains in the patient with CKD and assist in treatment.

In addition, since patients with $\mathrm{CKD}$ are routinely treated with many drugs, a number of which also rely upon the OAT system of the kidney for uptake and elimination $(10,31)$, this finding suggests that, 
in the setting of progressive kidney failure, competition for OAT-mediated transport between drugs and metabolites, as well as between metabolites, could have significant consequences in regards to plasma levels of uremic toxins. This is particularly important when one considers that probenecid is, in fact, a wellknown drug that has long been used to treat gout and to prolong the half-life of antibiotics (67). In addition, several of these probenecid-sensitive uremic solutes possess signaling capacity. For example, indoxyl sulfate can signal through the aryl hydrocarbon receptor (AHR), the activation of which is associated with a number of adverse conditions, including vascular inflammation, the production of reactive oxygen species, and cardiotoxicity (68-70). Kynurenic acid, which has a role in signaling in the central nervous system, is also an AHR ligand, as well as a ligand of the G protein-coupled receptor 35 (GPR35), which has also been associated with a number of diseases, including hypertension and heart failure $(71,72)$. In this regard, it is worth noting that the metabolic derangements of uremia reflect aberrant functioning of normal interorgan and interorganismal remote sensing and signaling, involving, among other ADME genes, multispecific transporters, such as the OATs $(31,73-77)$.

Consistent with general concepts of the remote sensing and signaling hypothesis, many of the uremic solutes are metabolic byproducts or intermediate molecules derived from endogenous cellular metabolism involving multiple tissues/organs, but many are also derived from "dysbiotic" gut microbiota. Thus, there is altered remote interorgan as well as altered interorganismal communication of metabolites, signaling molecules, and antioxidants transported by "drug" transporters (e.g., OAT1, OAT3) and acted upon by drug metabolizing enzymes (e.g., cyps, sulfotransferases). For example, almost $30 \%$ of the probenecid-sensitive uremic solutes have been shown to be derived from the action of the colonic microbiota from several studies $(47-51,53-62,78)$. Once again, since these metabolites would contribute to metabolite-metabolite and/or metabolite-drug competition at the level of the transporter (as might be argued given the effects of probenecid, a drug that competitively inhibits OAT function) $(67,79)$, their increased concentration in the plasma could not only potentially adversely affect residual tubular secretion in the failing kidney but alter systemic metabolism and, by entering other tissues (perhaps by OATs, OATPs and MRPs), deleteriously alter the function of other organs. Thus, it would seem that strategies aimed at limiting the generation of these uremic toxins early in the development of CKD could have beneficial effects, such as limiting the dietary source or treating with an intestinal absorbent such as AST-120 (51, 80-82). Moreover, since CKD and increasing levels of uremic toxins also can lead to gut dysbiosis (reflecting aberrant bidirectional interorganismal remote communication) and the generation of additional uremic solutes that could accelerate the progression of $\operatorname{CKD}(37,83)$, it is clear that limiting the accumulation of these uremic solutes/toxins early in the process could abort this vicious cycle and preserve residual tubular secretory activity.

\section{Methods}

STN. Animals were housed for 1 week before surgery for acclimation after shipping with free access to water and standard rat chow. The surgical procedure was then performed with sterile technique on a temperature-controlled surgical table and under anesthesia with pentobarbital sodium (50 mg/kg i.p.) (32, 84-87). Briefly, a small incision (1.5-cm long) was made in the abdomen and, following ligation of the renal artery and vein, the right kidney was removed. The left renal artery was then exposed and 2 of its branches were ligated with 4-0 silk suture. Exposed tissue was then replaced, and the incision closed. Rats were kept warm with a heating pad until ambulatory and administered a dose of buprenorphine analgesic. The animals were then housed for an additional 2 weeks after surgery with free access to water and standard rat chow (Envigo).

Treatment with probenecid. Following 2 weeks of recovery, animals were given a single i.p. injection of either $200 \mathrm{mg} / \mathrm{kg}$ water-soluble probenecid (Invitrogen) $(0.02 \mathrm{mg} / \mu \mathrm{L}$ in PBS [10 $\mu \mathrm{L} / \mathrm{g}$ of body weight]) or PBS (sham-treated control). After 2 hours of probenecid exposure, the animals were sacrificed, blood was collected for plasma/serum isolation, and individual unpooled serum samples were stored at $-80^{\circ} \mathrm{C}$ until used (33).

Measurement of PAH and inulin clearances. As previously described $(87,88)$, measurements of renal function were made under Inactin (thiobutabarbital sodium salt hydrate; T133; MilliporeSigma) anesthesia (100 $\mathrm{mg} / \mathrm{kg}$ i.p.). Briefly, after placing a tracheostomy tube (PE-240) to secure the airway, catheters were placed in the left jugular vein (PE-50) for fluid and drug administration, the femoral artery (PE-50) for blood pressure monitoring and blood sampling, and the urinary bladder (PE-50) for urine collection. Animals were maintained on a servo-controlled heating table with rectal temperature probe.

PAH clearance. PAH, prepared by diluting $250 \mu \mathrm{Ci}\left[{ }^{14} \mathrm{C}\right] \mathrm{PAH}$ (Perkin Elmer) in $50 \mathrm{ml}$ sterile saline, was infused at the rate of $1.5 \mathrm{ml} / \mathrm{h}$ throughout the experiment. After 60 minutes of stabilization, a 2-period 
study was performed, with period 1 consisting of saline infusion at $2 \mathrm{ml} / \mathrm{h}$ and period 2 consisting of probenecid infusion $(200 \mathrm{mg} / \mathrm{kg}$ ) at $2 \mathrm{ml} / \mathrm{h}$. Urine was collected for the duration of each period (60 minutes), and plasma samples were collected at the end of each period. For GFR measurements, inulin clearances were performed simultaneously during both periods, with the infusion of $\left[{ }^{3} \mathrm{H}\right]$ Inulin at $2 \mathrm{ml} / \mathrm{h}$. PAH and inulin clearances were calculated by the following equations: $\mathrm{PAH}$ clearance $(\mathrm{ml} / \mathrm{min})=$ (urine $\mathrm{PAH}$ concentration $\times$ urine volume $) /($ plasma PAH concentration $\times$ time $)$ and inulin clearance $(\mathrm{ml} / \mathrm{min})=($ urine inulin concentration $\times$ urine volume $) /($ plasma inulin concentration $\times$ time $)$.

Metabolomics analyses. Plasma samples were shipped frozen on dry-ice to Metabolon where they were subjected to ultra-high performance liquid chromatography/tandem accurate mass spectrometry, allowing for accurate relative quantification of 668 metabolites of known identity $(21,33)$.

Compound identification, quantification, data curation, and statistics. The present data set comprises a total of 668 metabolites of known identity in rat plasma, which were identified as previously described (21, 33). Briefly, ion features (e.g., retention time, molecular weight $[\mathrm{m} / \mathrm{z}]$, preferred adducts, and in-source fragments as well as associated MS spectra) were compared between the samples and a chemical standard reference library using software developed at Metabolon $(89,90)$.

The individual 668 metabolites were also grouped into 8 biochemical superpathways as defined by Metabolon $(21,22,33,89,90)$. The 8 superpathways (i.e., amino acids, carbohydrates, cofactors and vitamins, energy, lipids, nucleotides, peptides, and xenobiotics) were further divided into subpathways into which the individual metabolites were then sorted. For example, the amino acids superpathway comprises 15 subpathways (i.e., metabolism of (glycine, serine, and threonine; alanine and aspartate; glutamate; histidine; lysine; phenylalanine; tyrosine; tryptophan; leucine, isoleucine, and valine; methionine, cysteine, SAM, and taurine; arginine and proline; creatine; polyamine; guanidino and acetamido; as well as glutathione) into which 175 individual metabolites are sorted. On the Metabolon website, "pathway enrichment" is described as a value representing "the number of significantly altered metabolites relative to all detected compounds in a subpathway, compared to the total number of statistically significantly different compounds relative to all detected compounds in the study" (94). The value is calculated by the following formula: enrichment value = $(k / m) /(n-k / N-m)$, where $k$ denotes the number of significant metabolites in the pathway; $m$ denotes number of metabolites in the pathway; $n$ represents the total number of significant metabolites on platform; and $N$ represents the total number of metabolites on platform (94). Thus, in this study, the enrichment values shown in Table 2 were calculated using these parameters, with the exception that the metabolites used for the calculation were limited to the uremic toxins that are present on the platform (Supplemental Table 1).

Data collection from previous metabolomics analyses. The study involved the comparison of the metabolomics data in this study with that from previous metabolomics analyses (15, 16, 20-22, 33, 43-59).

Comparison of Oat1-KO and Oat3-KO metabolomics with data from STN rats treated with probenecid. Several metabolomics analyses, both targeted and untargeted, of the serum and/or urine of the Oat1-KO and Oat3KO mice have been performed $(15,16,20-22,33,43-45)$. While the metabolomics were performed using a different species and different facilities using differing methodologies (e.g., the Oat3-KO metabolomics analyses included data from both male and female $\mathrm{K} \mathrm{O}$ mice), relative changes in metabolite concentrations can be compared for identified compounds across the platforms. Thus, data from these previous metabolomics analyses were compared with the current metabolomics data from the STN rat to identify those probenecid-sensitive uremic solutes that were also increased in the plasma of the Oat-KO animals.

Comparison to colon-derived endogenous metabolites. As described above for the $\mathrm{KO}$ mice, several metabolomics analyses have been performed on serum and/or urine collected from patients with CKD (44-59), and data from these studies, which also used differing methodologies and were from a different species, were used here. Metabolites identified in the current study were overlapped with these human metabolomics analyses, and relative changes in plasma concentration of those metabolites common to the various platforms were compared.

Statistics. For glomerular filtration and tubular secretion, statistical significance was determined using a 2-tailed Student's $t$ test, and a difference between experimental groups was considered statistically significant when the $P$ value was less than $0.05(P \leq 0.05)$.

For metabolomics analyses with STN, following normalization to volume, log transformation, and the imputation of any missing values with the minimum observed value for each compound, a 2-tailed Welch's 2-sample $t$ test was used to identify biochemicals that differed significantly between the experimental groups (i.e., sham treated vs. STN and STN vs. STN + probenecid) $(89,90)$. A metabolite was considered to be 
statistically different between groups when the $P$ value was less than $0.05(P \leq 0.05)$ and was considered to be approaching a significant difference between groups if the $P$ value was greater than 0.05 but less than or equal to $0.1(0.05<P \leq 0.1)$.

As described previously $(21,33,91)$, the $P$ values in the metabolomics analyses of the Oat-KO animals were calculated using 2-way ANOVA testing. A $P$ value less than or equal to $0.05(P \leq 0.05)$ indicated that a metabolite was statistically different between groups, while a $P$ value greater than 0.05 but less than or equal to $0.1(0.05<P<0.1)$ indicated that a metabolite was approaching a significant difference.

Study approval. All experimentation involving the use of animals was conducted according to the National Institutes of Health's Guide for the Care and Use of Laboratory Animals (National Academies Press, 2011). The experiments were approved by the Veterans Affairs San Diego Healthcare System Institutional Animal Care and Use Committee. All animal experiments were conducted in male Wistar rats with body weight of 300-350 g (Harlan).

\section{Author contributions}

PS performed most of the wet-lab experimental aspects of the study and edited the manuscript. KTB performed most of the data analyses, wrote the manuscript, and prepared the figures and tables. SKN conceived and supervised the project, edited the manuscript, and independently confirmed aspects of the analysis.

\section{Acknowledgments}

This work was partly supported by US National Institutes of Health grants (National Institute of Diabetes, Digestive and Kidney Diseases grant R01DK109392 and Eunice K. Shriver National Institute of Child Health and Human Development grant U54HD090259 [to SKN]; R01DK107852 [to PS]), a Veterans Affairs Merit Award (BX002175 to PS), and resources from the UAB-UCSD O'Brien Center (National Institutes of Health grant P30-DK079337).

Address correspondence to: Sanjay K. Nigam, University of California, San Diego, 9500 Gilman Drive, MC0693, La Jolla, California 92093, USA. Email: snigam@ucsd.edu.

1. Vanholder R, et al. A bench to bedside view of uremic toxins. J Am Soc Nephrol. 2008;19(5):863-870.

2. Leong SC, Sao JN, Taussig A, Plummer NS, Meyer TW, Sirich TL. Residual function effectively controls plasma concentrations of secreted solutes in patients on twice weekly hemodialysis. J Am Soc Nephrol. 2018;29(7):1992-1999.

3. Lowenstein J, Grantham JJ. Residual renal function: a paradigm shift. Kidney Int. 2017;91(3):561-565.

4. Shafi T, Mullangi S, Toth-Manikowski SM, Hwang S, Michels WM. Residual kidney function: implications in the era of personalized medicine. Semin Dial. 2017;30(3):241-245.

5. Jansen J, Jankowski J, Gajjala PR, Wetzels JFM, Masereeuw R. Disposition and clinical implications of protein-bound uremic toxins. Clin Sci. 2017;131(14):1631-1647.

6. Lowenstein J, Grantham JJ. The rebirth of interest in renal tubular function. Am J Physiol Renal Physiol. 2016;310(11):F1351-F1355.

7. Marquez IO, et al. Contribution of residual function to removal of protein-bound solutes in hemodialysis. Clin J Am Soc Nephrol. 2011;6(2):290-296.

8. Wang K, Kestenbaum B. Proximal tubular secretory clearance: a neglected partner of kidney function. Clin J Am Soc Nephrol. 2018;13(8):1291-1296.

9. Masereeuw R, et al. The kidney and uremic toxin removal: glomerulus or tubule? Semin Nephrol. 2014;34(2):191-208

10. Nigam SK, Wu W, Bush KT, Hoenig MP, Blantz RC, Bhatnagar V. Handling of drugs, metabolites, and uremic toxins by kidney proximal tubule drug transporters. Clin J Am Soc Nephrol. 2015;10(11):2039-2049.

11. Sirich TL, Aronov PA, Plummer NS, Hostetter TH, Meyer TW. Numerous protein-bound solutes are cleared by the kidney with high efficiency. Kidney Int. 2013;84(3):585-590.

12. Sirich TL, Funk BA, Plummer NS, Hostetter TH, Meyer TW. Prominent accumulation in hemodialysis patients of solutes normally cleared by tubular secretion. J Am Soc Nephrol. 2014;25(3):615-622.

13. Suchy-Dicey AM, et al. Tubular secretion in CKD. J Am Soc Nephrol. 2016;27(7):2148-2155.

14. Nigam SK. What do drug transporters really do? Nat Rev Drug Discov. 2015;14(1):29-44.

15. Eraly SA, et al. Decreased renal organic anion secretion and plasma accumulation of endogenous organic anions in OAT1 knock-out mice. J Biol Chem. 2006;281(8):5072-5083.

16. Liu HC, et al. An organic anion transporter 1 (OAT1)-centered metabolic network. J Biol Chem. 2016;291(37):19474-19486.

17. Nigam SK. The SLC22 transporter family: a paradigm for the impact of drug transporters on metabolic pathways, signaling, and Disease. Annu Rev Pharmacol Toxicol. 2018;58:663-687.

18. Nigam SK, et al. The organic anion transporter (OAT) family: a systems biology perspective. Physiol Rev. 2015;95(1):83-123

19. Sweet DH, Miller DS, Pritchard JB, Fujiwara Y, Beier DR, Nigam SK. Impaired organic anion transport in kidney and choroid plexus of organic anion transporter 3 (Oat3 (Slc22a8)) knockout mice. J Biol Chem. 2002;277(30):26934-26943.

20. Wikoff WR, Nagle MA, Kouznetsova VL, Tsigelny IF, Nigam SK. Untargeted metabolomics identifies enterobiome metabolites and putative uremic toxins as substrates of organic anion transporter 1 (Oat1). J Proteome Res. 2011;10(6):2842-2851. 
21. Wu W, Bush KT, Nigam SK. Key role for the organic anion transporters, OAT1 and OAT3, in the in vivo handling of uremic toxins and solutes. Sci Rep. 2017;7(1):4939.

22. Wu W, et al. Multispecific drug transporter Slc22a8 (Oat3) regulates multiple metabolic and signaling pathways. Drug Metab Dispos. 2013;41(10):1825-1834.

23. Ahn SY, Nigam SK. Toward a systems level understanding of organic anion and other multispecific drug transporters: a remote sensing and signaling hypothesis. Mol Pharmacol. 2009;76(3):481-490.

24. Wu W, Dnyanmote AV, Nigam SK. Remote communication through solute carriers and ATP binding cassette drug transporter pathways: an update on the remote sensing and signaling hypothesis. Mol Pharmacol. 2011;79(5):795-805.

25. Motojima M, Hosokawa A, Yamato H, Muraki T, Yoshioka T. Uraemic toxins induce proximal tubular injury via organic anion transporter 1-mediated uptake. Br J Pharmacol. 2002;135(2):555-563.

26. Motojima M, Hosokawa A, Yamato H, Muraki T, Yoshioka T. Uremic toxins of organic anions up-regulate PAI-1 expression by induction of NF-kappaB and free radical in proximal tubular cells. Kidney Int. 2003;63(5):1671-1680.

27. Fujii H, Goto S, Fukagawa M. Role of uremic toxins for kidney, cardiovascular, and bone dysfunction. Toxins (Basel). 2018;10(5):E202.

28. Saito H. Pathophysiological regulation of renal SLC22A organic ion transporters in acute kidney injury: pharmacological and toxicological implications. Pharmacol Ther. 2010;125(1):79-91.

29. Mónica Torres A, Mac Laughlin M, Muller A, Brandoni A, Anzai N, Endou H. Altered renal elimination of organic anions in rats with chronic renal failure. Biochim Biophys Acta. 2005;1740(1):29-37.

30. Torres AM, Dnyanmote AV, Bush KT, Wu W, Nigam SK. Deletion of multispecific organic anion transporter Oat1/Slc22a6 protects against mercury-induced kidney injury. J Biol Chem. 2011;286(30):26391-26395.

31. Nigam SK, Bush KT. Uraemic syndrome of chronic kidney disease: altered remote sensing and signalling. Nat Rev Nephrol. 2019;15(5):301-316.

32. Singh P, Deng A, Blantz RC, Thomson SC. Unexpected effect of angiotensin AT1 receptor blockade on tubuloglomerular feedback in early subtotal nephrectomy. Am J Physiol Renal Physiol. 2009;296(5):F1158-F1165.

33. Bush KT, Wu W, Lun C, Nigam SK. The drug transporter OAT3 (SLC22A8) and endogenous metabolite communication via the gut-liver-kidney axis. J Biol Chem. 2017;292(38):15789-15803.

34. Maeda K, et al. Inhibitory effects of p-aminohippurate and probenecid on the renal clearance of adefovir and benzylpenicillin as probe drugs for organic anion transporter (OAT) 1 and OAT3 in humans. Eur J Pharm Sci. 2014;59:94-103.

35. Sweeney DE, Vallon V, Rieg T, Wu W, Gallegos TF, Nigam SK. Functional maturation of drug transporters in the developing, neonatal, and postnatal kidney. Mol Pharmacol. 2011;80(1):147-154.

36. Duranton F, et al. Normal and pathologic concentrations of uremic toxins. J Am Soc Nephrol. 2012;23(7):1258-1270.

37. Koppe L, Fouque D, Soulage CO. Metabolic abnormalities in diabetes and kidney disease: role of uremic toxins. Curr Diab Rep. 2018;18(10):97.

38. Hagos FT, et al. Probenecid, an organic anion transporter 1 and 3 inhibitor, increases plasma and brain exposure of $\mathrm{N}$-acetylcysteine. Xenobiotica. 2017;47(4):346-353.

39. Kikuchi R, Kusuhara H, Sugiyama D, Sugiyama Y. Contribution of organic anion transporter 3 (S1c22a8) to the elimination of p-aminohippuric acid and benzylpenicillin across the blood-brain barrier. J Pharmacol Exp Ther. 2003;306(1):51-58.

40. Minematsu T, Hashimoto T, Aoki T, Usui T, Kamimura H. Role of organic anion transporters in the pharmacokinetics of zonampanel, an alpha-amino-3-hydroxy-5-methylisoxazole-4-propionate receptor antagonist, in rats. Drug Metab Dispos. 2008;36(8):1496-1504.

41. Kopple JD. Phenylalanine and tyrosine metabolism in chronic kidney failure. J Nutr. 2007;137(6):1586S-1590S

42. Duranton F, et al. Plasma and urinary amino acid metabolomic profiling in patients with different levels of kidney function. Clin J Am Soc Nephrol. 2014;9(1):37-45.

43. Eraly SA, et al. Multiple organic anion transporters contribute to net renal excretion of uric acid. Physiol Genomics. 2008;33(2):180-192.

44. Vallon V, et al. A role for the organic anion transporter OAT3 in renal creatinine secretion in mice. Am J Physiol Renal Physiol. 2012;302(10):F1293-F1299.

45. Vallon V, et al. Organic anion transporter 3 contributes to the regulation of blood pressure. J Am Soc Nephrol. 2008;19(9):1732-1740.

46. Armani RG, Ramezani A, Yasir A, Sharama S, Canziani MEF, Raj DS. Gut microbiome in chronic kidney disease. Curr Hypertens Rep. 2017;19(4):29.

47. Aronov PA, et al. Colonic contribution to uremic solutes. J Am Soc Nephrol. 2011;22(9):1769-1776.

48. Claus SP, et al. Systemic multicompartmental effects of the gut microbiome on mouse metabolic phenotypes. Mol Syst Biol. 2008;4:219.

49. González-Barrio R, Borges G, Mullen W, Crozier A. Bioavailability of anthocyanins and ellagitannins following consumption of raspberries by healthy humans and subjects with an ileostomy. J Agric Food Chem. 2010;58(7):3933-3939.

50. Jaganath IB, Mullen W, Edwards CA, Crozier A. The relative contribution of the small and large intestine to the absorption and metabolism of rutin in man. Free Radic Res. 2006;40(10):1035-1046.

51. Kikuchi K, Itoh Y, Tateoka R, Ezawa A, Murakami K, Niwa T. Metabolomic analysis of uremic toxins by liquid chromatography/electrospray ionization-tandem mass spectrometry. J Chromatogr B Analyt Technol Biomed Life Sci. 2010;878(20):1662-1668.

52. Bhowmik SK, An JH, Lee SH, Jung BH. Alteration of bile acid metabolism in pseudo germ-free rats [corrected]. Arch Pharm Res. 2012;35(11):1969-1977.

53. Nicholls AW, Mortishire-Smith RJ, Nicholson JK. NMR spectroscopic-based metabonomic studies of urinary metabolite variation in acclimatizing germ-free rats. Chem Res Toxicol. 2003;16(11):1395-1404.

54. Stalmach A, Edwards CA, Wightman JD, Crozier A. Colonic catabolism of dietary phenolic and polyphenolic compounds from Concord grape juice. Food Funct. 2013;4(1):52-62.

55. Wikoff WR, et al. Metabolomics analysis reveals large effects of gut microflora on mammalian blood metabolites. Proc Natl Acad Sci USA. 2009;106(10):3698-3703.

56. Yap IK, et al. Metabonomic and microbiological analysis of the dynamic effect of vancomycin-induced gut microbiota modifi- 
cation in the mouse. J Proteome Res. 2008;7(9):3718-3728.

57. Kikuchi M, Ueno M, Itoh Y, Suda W, Hattori M. Uremic toxin-producing gut microbiota in rats with chronic kidney disease. Nephron. 2017;135(1):51-60.

58. Mair RD, Sirich TL, Plummer NS, Meyer TW. Characteristics of colon-derived uremic solutes. Clin J Am Soc Nephrol. 2018;13(9):1398-1404.

59. Mishima E, et al. Evaluation of the impact of gut microbiota on uremic solute accumulation by a CE-TOFMS-based metabolomics approach. Kidney Int. 2017;92(3):634-645.

60. Nazzal L, et al. Microbiome perturbation by oral vancomycin reduces plasma concentration of two gut-derived uremic solutes, indoxyl sulfate and p-cresyl sulfate, in end-stage renal disease. Nephrol Dial Transplant. 2017;32(11):1809-1817.

61. Tanaka H, Sirich TL, Plummer NS, Weaver DS, Meyer TW. An enlarged profile of uremic solutes. PLoS ONE. 2015;10(8):e0135657.

62. Tang WH, et al. Intestinal microbial metabolism of phosphatidylcholine and cardiovascular risk. NEngl J Med. 2013;368(17):1575-1584.

63. Lopez-Nieto CE, You G, Bush KT, Barros EJ, Beier DR, Nigam SK. Molecular cloning and characterization of NKT, a gene product related to the organic cation transporter family that is almost exclusively expressed in the kidney. J Biol Chem. 1997;272(10):6471-6478.

64. Brandoni A, Torres AM. Altered renal expression of relevant clinical drug transporters in different models of acute uremia in rats. Role of urea levels. Cell Physiol Biochem. 2015;36(3):907-916.

65. Di Giusto G, Anzai N, Endou H, Torres AM. Elimination of organic anions in response to an early stage of renal ischemia-reperfusion in the rat: role of basolateral plasma membrane transporters and cortical renal blood flow. Pharmacology. 2008;81(2):127-136

66. Follman KE, Dave RA, Morris ME. Effects of renal impairment on transporter-mediated renal reabsorption of drugs and renal drug-drug interactions A simulation-based study. Biopharm Drug Dispos. 2018;39(4):218-231.

67. Robbins N, Koch SE, Tranter M, Rubinstein J. The history and future of probenecid. Cardiovasc Toxicol. 2012;12(1):1-9.

68. Kamiński T, Michałowska M, Pawlak D. Aryl hydrocarbon receptor (AhR) and its endogenous agonist - indoxyl sulfate in chronic kidney disease. Postepy Hig Med Dosw (Online). 2017;71(0):624-632.

69. Leong SC, Sirich TL. Indoxyl sulfate-Review of toxicity and therapeutic strategies. Toxins (Basel). 2016;8(12):E358.

70. Schroeder JC, et al. The uremic toxin 3-indoxyl sulfate is a potent endogenous agonist for the human aryl hydrocarbon receptor. Biochemistry. 2010;49(2):393-400.

71. Agudelo LZ, et al. Kynurenic acid and Gpr35 regulate adipose tissue energy homeostasis and inflammation. Cell Metab. 2018;27(2):378-392.e5.

72. Wang J, et al. Kynurenic acid as a ligand for orphan G protein-coupled receptor GPR35. J Biol Chem. 2006;281(31):22021-22028.

73. Bhatnagar V, et al. Analysis of ABCG2 and other urate transporters in uric acid homeostasis in chronic kidney disease: potential role of remote sensing and signaling. Clin Kidney J. 2016;9(3):444-453.

74. Liu B, Luo F, Luo X, Duan S, Gong Z, Peng J. Metabolic enzyme system and transport pathways in chronic kidney diseases. Curr Drug Metab. 2018;19(7):568-576.

75. Naud J, Michaud J, Leblond FA, Lefrancois S, Bonnardeaux A, Pichette V. Effects of chronic renal failure on liver drug transporters. Drug Metab Dispos. 2008;36(1):124-128.

76. Naud J, Nolin TD, Leblond FA, Pichette V. Current understanding of drug disposition in kidney disease. J Clin Pharmacol. 2012;52(1 Suppl):10S-22S

77. Takada T, et al. Identification of ABCG2 as an exporter of uremic toxin indoxyl sulfate in mice and as a crucial factor influencing CKD progression. Sci Rep. 2018;8(1):11147.

78. Lee SH, An JH, Park HM, Jung BH. Investigation of endogenous metabolic changes in the urine of pseudo germ-free rats using a metabolomic approach. J Chromatogr B Analyt Technol Biomed Life Sci. 2012;887-888:8-18.

79. Cunningham RF, Israili ZH, Dayton PG. Clinical pharmacokinetics of probenecid. Clin Pharmacokinet. 1981;6(2):135-151.

80. Lau WL, Savoj J, Nakata MB, Vaziri ND. Altered microbiome in chronic kidney disease: systemic effects of gut-derived uremic toxins. Clin Sci. 2018;132(5):509-522.

81. Niwa T. Targeting protein-bound uremic toxins in chronic kidney disease. Expert Opin Ther Targets. 2013;17(11):1287-1301.

82. Schulman G, et al. Randomized placebo-controlled EPPIC trials of AST-120 in CKD. J Am Soc Nephrol. 2015;26(7):1732-1746.

83. Evenepoel P, Poesen R, Meijers B. The gut-kidney axis. Pediatr Nephrol. 2017;32(11):2005-2014.

84. Li H, et al. Interactions between HIF-1 $\alpha$ and AMPK in the regulation of cellular hypoxia adaptation in chronic kidney disease. Am J Physiol Renal Physiol. 2015;309(5):F414-F428.

85. Singh P, Blantz RC, Rosenberger C, Gabbai FB, Schoeb TR, Thomson SC. Aberrant tubuloglomerular feedback and HIF-1 $\alpha$ confer resistance to ischemia after subtotal nephrectomy. J Am Soc Nephrol. 2012;23(3):483-493.

86. Singh P, Thomson SC. Salt sensitivity of tubuloglomerular feedback in the early remnant kidney. Am J Physiol Renal Physiol. 2014;306(2):F172-F180.

87. Thomas JL, et al. Hypoxia-inducible factor-1 $\alpha$ activation improves renal oxygenation and mitochondrial function in early chronic kidney disease. Am J Physiol Renal Physiol. 2017;313(2):F282-F290.

88. Schneider R, et al. Downregulation of organic anion transporters OAT1 and OAT3 correlates with impaired secretion of para-aminohippurate after ischemic acute renal failure in rats. Am J Physiol Renal Physiol. 2007;292(5):F1599-F1605.

89. Evans AM, DeHaven CD, Barrett T, Mitchell M, Milgram E. Integrated, nontargeted ultrahigh performance liquid chromatography/electrospray ionization tandem mass spectrometry platform for the identification and relative quantification of the small-molecule complement of biological systems. Anal Chem. 2009;81(16):6656-6667.

90. Ohta T, et al. Untargeted metabolomic profiling as an evaluative tool of fenofibrate-induced toxicology in Fischer 344 male rats. Toxicol Pathol. 2009;37(4):521-535.

91. Nigam AK, et al. Unique metabolite preferences of the drug transporters OAT1 and OAT3 analyzed by machine learning. $J$ Biol Chem. 2020;295(7):1829-1842.

92. Spitzer M, Wildenhain J, Rappsilber J, Tyers M. BoxPlotR: a web tool for generation of box plots. Nat Methods. 2014;11(2):121-122. 
93. Kikuchi K, Itoh Y, Tateoka R, Ezawa A, Murakami K, Niwa T. Metabolomic search for uremic toxins as indicators of the effect of an oral sorbent AST-120 by liquid chromatography/tandem mass spectrometry. J Chromatogr B Analyt Technol Biomed Life Sci. 2010;878(29):2997-3002.

94. Metabolon client portal. https://mportal-static.s3.amazonaws.com/Help/FAQ.pdf. Accessed March 31, 2020.

95. Beebe K, Pappan K. Metabolomics-a key technology for microbiome research. https://www.metabolon.com/application/ files/5115/0904/9732/Metabolomics_-_A_Key_Technology_for_Microbiome_Research_eBook.pdf. Accessed April 1, 2020. 